\title{
Band-splitting of coronal and interplanetary type II bursts
}

\section{Basic properties}

\author{
B. Vršnak ${ }^{1}$, H. Aurass ${ }^{2}$, J. Magdalenić ${ }^{3}$, and N. Gopalswamy ${ }^{4}$ \\ 1 Hvar Observatory, University of Zagreb, Kačićeva 26, 10000 Zagreb, Croatia \\ e-mail: bvrsnak@geodet.geof.hr \\ 2 Astrophysikalisches Institut Potsdam, An der Sternwarte 16, 14482 Potsdam, Germany \\ e-mail: haurass@aip.de \\ 3 Hvar Observatory, University of Zagreb, Kačićeva 26, HR - 10000 Zagreb, Croatia \\ e-mail: jasminam@operamail.com \\ 4 NASA GSFC, Greenbelt, Maryland, USA \\ e-mail: gopals@fugee.gsfc.nasa.gov
}

Received 31 May 2001 / Accepted 24 July 2001

\begin{abstract}
Patterns analogous to the band-splitting of metric type II bursts are found in a number of type II bursts observed in the dekameter-kilometer wavelength range. A similarity of morphological and frequency-time characteristics of two emission components are indicative of a common source. Relative frequency splits span in the range $\Delta f / f=0.05-0.6$. At radial distances between 2 and $4 R_{\odot}$ only small splits around 0.1 can be found. In the interplanetary space the relative split on average increases with the radial distance, whereas the inferred shock velocity decreases. In three events extrapolations of the split components point to the base and the peak of the jump in the local plasma frequency caused by the associated shock passage at $1 \mathrm{AU}$. This is suggestive of the plasma radiation from the regions upstream and downstream of the shock. Adopting this interpretation, one finds that the drop of $\Delta f / f$ at $2-4 R_{\odot}$ is congruent with the Alfvén velocity maximum expected there. The split increase and the velocity decrease at larger distances can be explained as a consequence of declining Alfvén speed in the interplanetary space.
\end{abstract}

Key words. Sun: radio radiation - MHD - shock waves - Sun: corona - interplanetary medium

\section{Introduction}

Slowly drifting, narrowband patterns observed in radio dynamic spectra, denoted as type II radio bursts (hereinafter t-II bursts), are signatures of MHD shock waves (Nelson \& Melrose 1985 and references therein). The metric wavelength t-II bursts, appearing typically at frequencies around or below $100 \mathrm{MHz}$, are caused by shocks traveling through the solar corona, whereas those recorded in the dekameter to kilometer wavelength range are excited by shocks traveling through the upper corona (UC) and interplanetary (IP) space.

Metric t-II bursts usually show the fundamental and harmonic emission band (hereinafter $F$ - and $H$-band, respectively), both frequently being split in two parallel lanes (cf. Nelson \& Melrose 1985). A number of interpretations was proposed regarding the band-splitting accounting for the magnetic, Doppler, and geometrical effects (see Krüger 1979 and references therein). Smerd et al. (1974)

Send offprint requests to: B. Vršnak, e-mail: bvrsnak@geodet.geof.hr proposed an explanation in terms of the emission from the upstream and downstream shock regions, revealing a potentially important tool that could provide an estimate of the coronal Alfvén speed and magnetic field.

Mann et al. (1995) and Mann et al. (1996) studied the relative instantaneous bandwidth in a number of metric t-II bursts. It was found that relative bandwidths range from 0.1 up to 0.7 with the distribution maximum around 0.3 , and that the values are positively correlated with the radio source velocity. Adopting the upstream/downstream interpretation the conclusion was drawn that shocks exciting t-II bursts are either weak supercritical quasi-parallel, or subcritical quasiperpendicular fast-mode MHD shocks.

UC/IP t-II bursts observed in the dekameter to kilometer wavelength range are characterized by weaker emission than their metric counterpart. Emission lanes are often torn in a series of patches. In contrast to metric tII bursts, the $F$-band is often stronger than the $H$-band (cf. Bougeret 1985, and references therein; see also 
Lengyel-Frey \& Stone 1989). Although the band-splitting was recognized in some events (Boischot et al. 1980; Cane et al. 1982) it was never considered as an important feature of UC/IP t-II bursts.

In this paper we compare morphological patterns recorded in coronal and UC/IP t-II bursts showing bandsplitting. Dynamic spectra of the Astrophysikalisches Institut Potsdam (Mann et al. 1992) and WAVES instrument onboard WIND spacecraft (Bougeret et al. 1995) are used, covering the wavelength range $800-40 \mathrm{MHz}$ and $14-0.02 \mathrm{MHz}$, respectively. First, in Sect. 2 we set some restrictive criteria to define the band-split patterns that are analyzed. In Sect. 3 basic properties of this phenomenon are investigated and the results are discussed in Sect. 4 .

\section{Observations}

\subsection{Morphological patterns: Criteria}

In the analysis only features possessing the following three characteristics are considered:

- two narrow-band emission lanes (and/or series of patches) show a synchronized frequency time evolution $(f(t))$;

- intensity fluctuations along the two lanes are similar;

- the frequency ratio of the lanes is different from the $H / F$ frequency ratio $f_{H} / f_{F}=2$.

The first two criteria are used to eliminate an accidental time association of two emission components. Namely, bearing in mind that shock waves encompass large coronal (or IP) regions it is quite possible that there are two or more radio sources located along the shock front. In that case multiple emission lanes would be recorded in the dynamic radio spectrum. Each of them would show a frequency drift governed by the corresponding radio source velocity along the density gradient. Furthermore, the intensity variation along the emission lane would be determined by the change of conditions along the radio source trajectory. It is not likely (although not impossible) that these two signatures are correlated in the case of two distant radio sources. So, the emission showing band-split that satisfies the first two criteria with a great probability can be attributed to a single radio source, or to two closely related sources. The third criterion is self-evident.

Inspecting the sample of 112 metric t-II bursts we found that 22 of them (20\%) show well defined bandsplitting satisfying the posed criteria. For the purposes of this paper we have chosen only six examples showing the best defined and most persistent band-splitting. In the case of UC/IP t-II bursts we found that 25 out of 139 events (17\%) show episodes of band-split emission matching the criteria. From this subsample eleven UC/IP t-II bursts most convenient for the following analysis were chosen.

We note that actually a somewhat larger fraction of events is indicative of band splitting. On one side there are events (especially in the metric wavelength range) whose dynamic spectra are too complex to allow for an unambiguous identification of band-split pairs and a reliable quantitative analysis. In such cases the spectrum is characterized most often by a "multi-lane" emission, probably coming from several sources (Weiss 1972). Yet, some fragments satisfy the posed criteria. On the other side, there are weak events whose emission patterns are sometimes indicative of band-splitting but a low signal to noise ratio again does not allow for a definite decision.

In Fig. 1a we present an example of a metric t-II burst showing a well defined band-splitting. The background subtraction was applied and then the emission elements of interest enhanced. This example was chosen particularly because it shows a fragmented, patchy emission pattern rather similar to UC/IP t-II bursts. We note that in fact most metric t-II bursts show such a property, unlike the events that are widely used as "typical" examples.

In the inset in Fig. 1a, a part of the spectrum is schematically drawn to particularly indicate the emission patches (thin full lines) that form two branches of the band-split pattern (bold lines). A similar behaviour of the frequency drift is obvious, and emission patterns along the two emission components show a similar morphology. The area covered by a "parasitic" emission, probably coming from another source on the shock front, is indicated by the thin dashed line. The latter feature also shows a bandsplit-like spectrum.

An example of UC t-II burst showing the bandsplitlike pattern in the $H$-band is exhibited in Fig. 1b. Note that the frequency is increasing along the $y$-axis (opposite to the example shown in Fig. 1a) as is usual in representing the long wavelength spectra. In the left panel the lines used for measurements are indicated. In the righthand panel the emission patches used to define the emission lanes are selectively saturated. (A similar procedure is performed in all of the spectra shown hereafter to mark legibly the considered elements.) Emission lanes show a synchronized $f(t)$ behaviour and similar intensity fluctuations. Note also the presence of "parasitic" emission as in the example shown in Fig. 1a.

In Fig. 1c an example of IP t-II burst is shown exposing the $F / H$ structure, and a prominent band-splitting of the $F$-band. As in the examples shown in Figs. 1a and b, a similarity of frequency drift and morphological patterns can be recognized. Again, some associated parasitic emission appears. The passage of the type II burst related shock, revealed by the local plasma frequency jump recorded on October 3, is shown in the inset.

Note that in Fig. 1c the local plasma frequency shows also a jump around $01 \mathrm{UT}$ on October 1, revealing the passage of an earlier shock. Weak t-II burst-like emission patches probably associated with this shock are marked at $\approx 60 \mathrm{kHz}$ on September 30 around 16 UT (left-below the indicated $F$-band). We stress that the extrapolation of this emission points to the peak of the associated local frequency jump, indicating that it is probably excited in the downstream region of the shock (see also Lengyel-Frey 1992). 

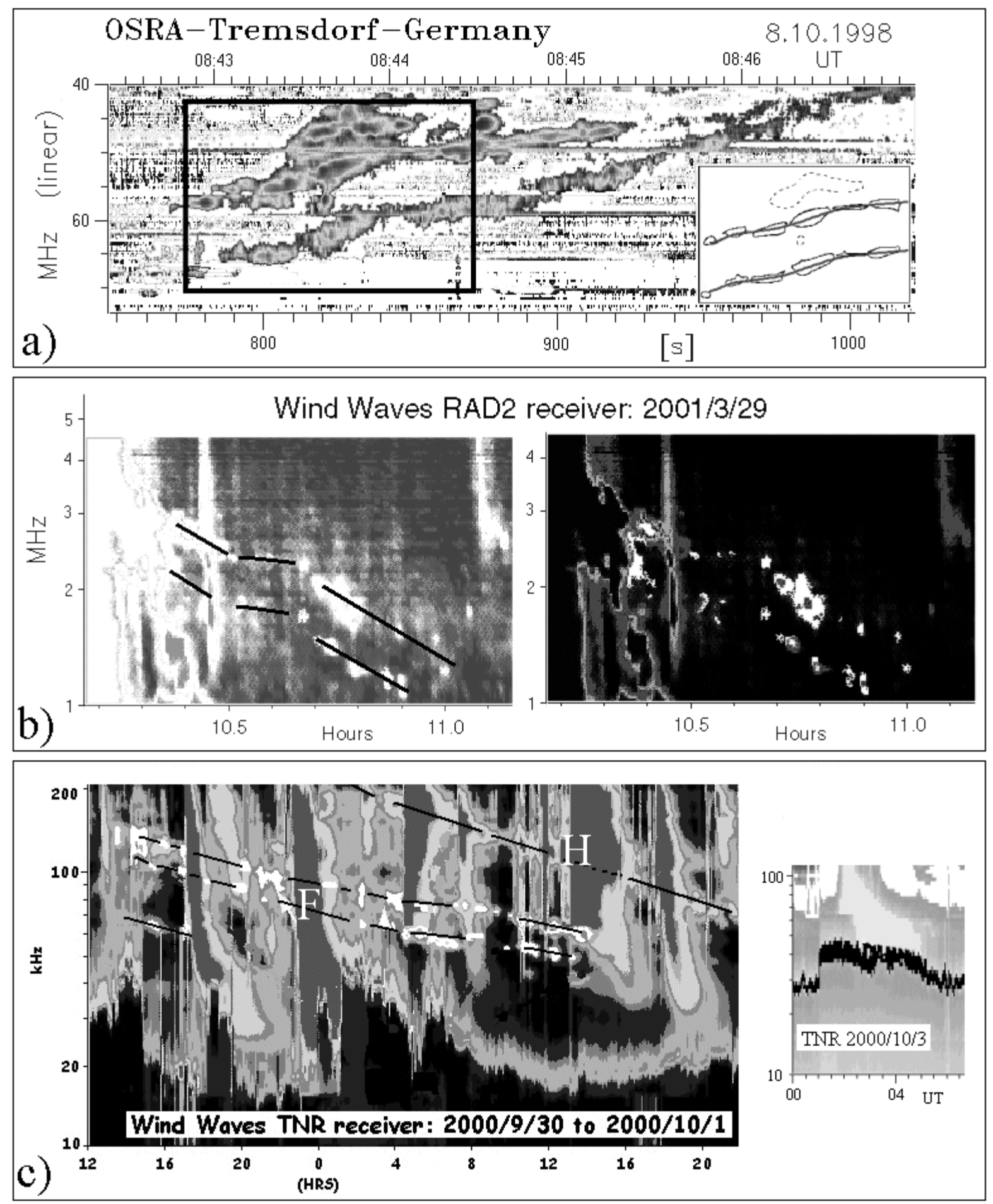

Fig. 1. a) A segment of the fundamental band of the coronal type II burst of October 8, 1998. Note that frequency is decreasing along the $y$-axis as is usual in representing the meter-wavelength spectra. The framed part of spectrum is schematically drawn in the inset. Emission probably coming from another source is marked by dashed line. b) UC type II burst of March $29,2001$. In the right-hand panel the patches forming a band-split pattern are shown enhanced. c) IP type II burst of September 30 to October 1, 2000 ( $F$-fundamental, $H$-harmonic). In the inset the jump of the local plasma frequency recorded at October 3 that was caused by the passage of the type II burst related shock is shown.

\subsection{Measurements}

In Fig. 2 an example of IP t-II burst is shown together with a schematic drawing of a band-split emission to describe the measurements. The instantaneous split at the moment $t$ is denoted as $\Delta f$. It is defined as the difference $\Delta f(t)=f_{1}(t)-f_{2}(t)$, where $f_{1}$ is the frequency of the lower frequency branch (LFB) emission and $f_{2}$ is the 

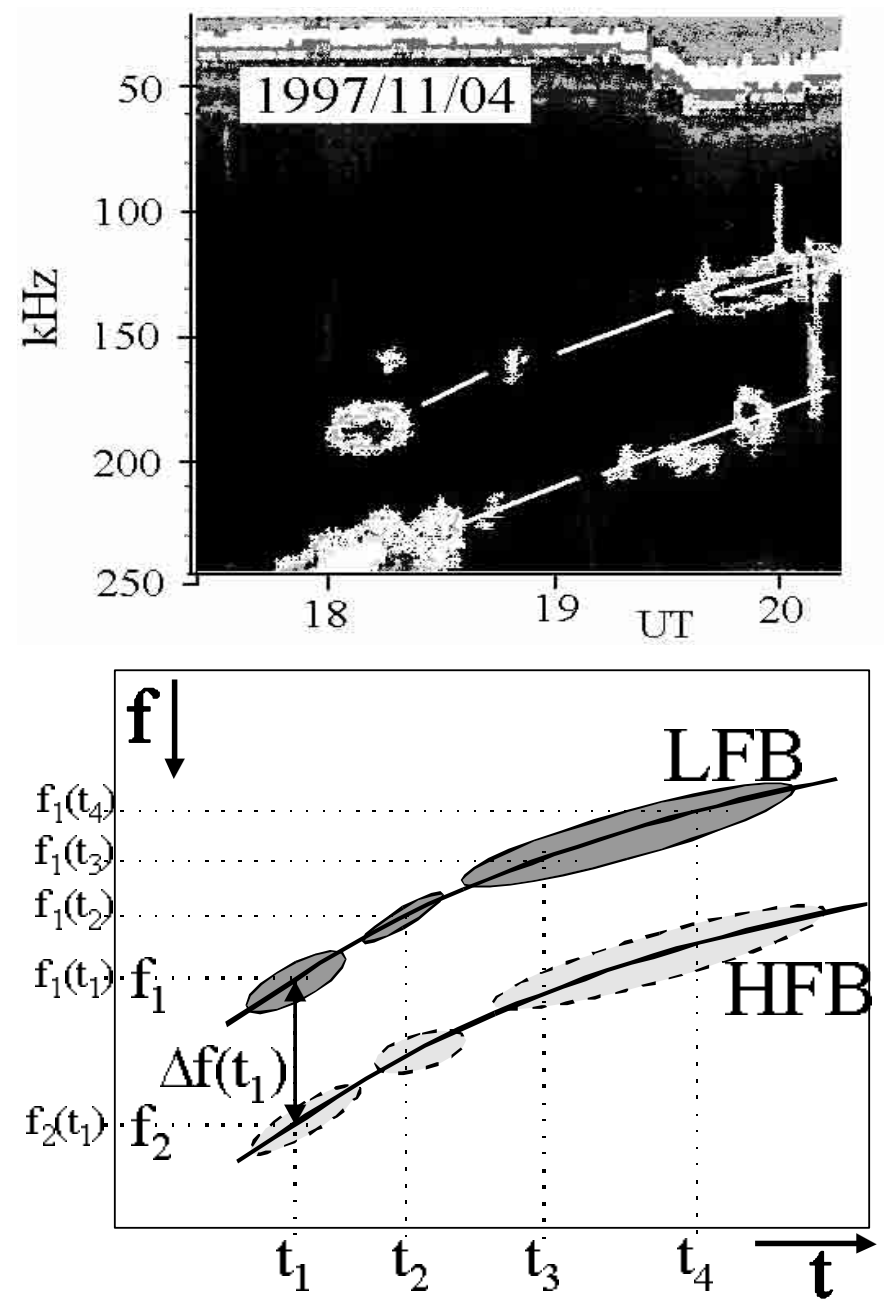

Fig. 2. A part of the dynamic spectrum of IP type II burst of November 3-6, 1997 (top) and schematic drawing of a band-split emission pattern (bottom). LFB denotes the lower frequency branch (darker gray), HFB denotes the higher frequency branch (lighter gray), and $\Delta f$ represents the instantaneous split.

frequency of the higher frequency branch (HFB). Note that the central frequency of an emission patch is measured. The relative instantaneous split is the ratio:

$B D W=\frac{\Delta f}{f_{1}}=\frac{f_{1}-f_{2}}{f_{1}}$.

The frequency drift at the moment $t$ is defined as $D_{f}(t)=$ $|\partial f / \partial t|_{t}$ and the relative frequency drift is:

$$
\left(\frac{D_{f}}{f}\right)_{t}=\left|\frac{1}{f} \frac{\partial f}{\partial t}\right|_{t}
$$

In practice it was determined using:

$$
\left(\frac{D_{f}}{f}\right)_{t}=\frac{\frac{f\left(t_{i+1}\right)-f\left(t_{i}\right)}{t_{i+1}-t_{i}}}{\frac{f\left(t_{i+1}\right)+f\left(t_{i}\right)}{2}},
$$

where $t=\left(t_{i+1}+t_{i}\right) / 2$ (see Fig. 2$)$. Let us note that $B D W$ and $D_{f} / f$ can be applied equally to the measurements at the $F$ - and $H$-band.

\subsection{Examples}

In Figs. 3a and 3b two examples of metric (i.e., coronal) t-II bursts are presented to illustrate different evolutionary characteristics. The lines used for measurements are drawn in the spectra. The obtained values of $D_{f} / f$ and $B D W$ are shown as a function of time $t$ in the middle and the rightmost panels, respectively.

The example in Fig. 3a shows an approximately constant $D_{f} / f$ and only a slight decrease of $B D W$. The example presented in Fig. 3 b shows a rapid decrease of $D_{f} / f$ and $B D W$ from relatively high values to the considerably lower ones. The final values are similar to those found in the previous example.

In Figs. 3c and d two IP t-II bursts observed at different wavelength ranges are presented for a comparison with the two metric t-II bursts shown in Figs. 3a and b. In general IP events show much lower relative frequency drifts $D_{f} / f$, whereas values of $B D W$ are comparable or slightly larger than in the coronal events. Note a similarity of band-splitting behaviour in Figs. 3a and d, although the first one appears in the 50-100 MHz range and the second one in the $50-100 \mathrm{kHz}$ range. Note that these frequencies correspond to distances of a few $R_{\odot}$ and some $100 R_{\odot}$, respectively. On the other hand, comparing Figs. 3a and c one finds also a remarkably similar morphology of the fine structure patterns.

\subsection{Relation to the in situ shocks}

Three of the studied IP t-II bursts were associated with the shocks detected by in situ measurements at $1 \mathrm{AU}$ (the events shown in Figs. 1c and 2, and the event of April 21-23, 1998). The dynamic spectra of these events, after being measured as described in Fig. 2, were plotted as the inverse frequency $1 / f$ versus time. Such a representation is convenient since the plasma density in the IP space decreases roughly as $n \propto R^{-2}$, and since $f \propto \sqrt{n}$, one finds $R \propto 1 / f$ (for details see Reiner et al. 2001).

The results are shown in Fig. 4 together with the linear least squares fits through LFBs and HFBs. In all three examples the extrapolation of the LFB fit points towards the foot of the associated $1 \mathrm{AU}$ shock signature. On the other hand, the extrapolations of the HFB fits are directed towards the peak of the shock signature. Such a relationship suggests that LFB and HFB emission can be interpreted as the plasma radiation from the upstream and downstream shock region, respectively.

\section{Properties of band-splitting}

The procedure illustrated in Fig. 3 was applied to six metric and eleven UC/IP t-II bursts. The results are summarized in Fig. 5. The number of data points per event varied between 4 and 10, depending on the duration of recognizable band-split emission. The radial distance $R(t)$ of the radio source was estimated from the frequency of the LFB emission $f_{1}(t)$ and will be expressed hereinafter in 

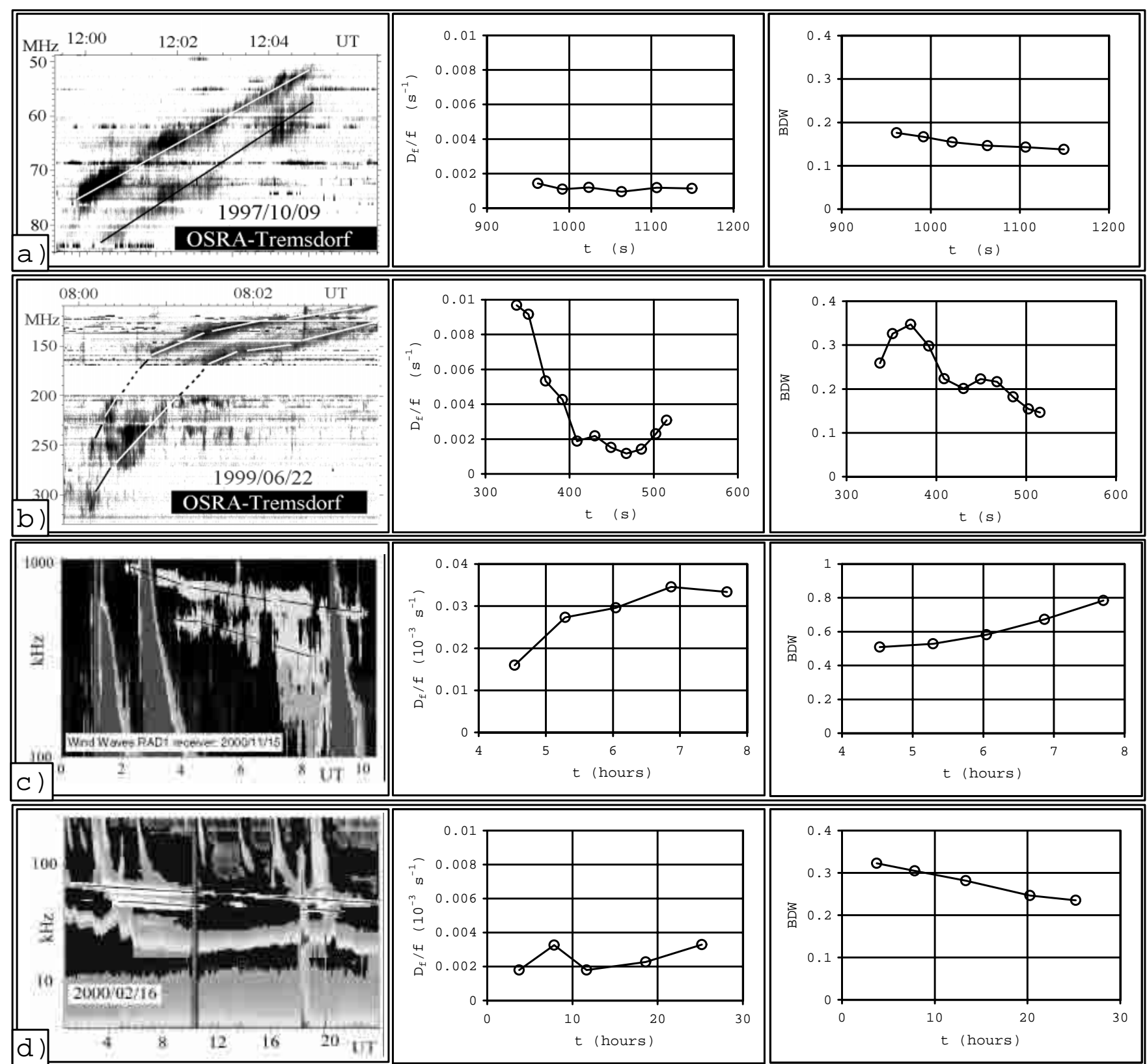

Fig. 3. Two examples of band-split type II emission observed in the metric wavelength range and two at long wavelengts are shown. The frequency axes are performed in the manner usual for metric and long wavelength spectra, respectively. The measured relative frequency drifts $\left(D_{f} / f\right)$ and relative splits $(B D W)$ are shown as a function of time $(t)$ in the middle and rightmost panels, respectively. a) September 9, 1997; b) June 22, 1999; c) November 11, 2000; d) February $16,2000$.

units of the solar radius $\left(R_{\odot}\right)$. In metric t-II bursts $R$ was obtained using the five-fold Saito coronal density model (Saito 1970), roughly corresponding to the two-fold Newkirk model (Newkirk 1961). For UC/IP t-II bursts the density model and the normalization procedure proposed by Leblanc et al. (1998) was adopted. The speed $v(t)$ of the radio source was inferred assuming the radial motion, presumably corresponding to the shock velocity (for a discussion see Vršnak et al. 2001).

In Fig. 5a the frequency drift $D_{f}(t)$ versus the frequency $f$ is shown. The graph reveals a remarkable correlation closely following the power law dependence (shown in the inset; the correlation coefficient $C=0.99$ ). Such a behaviour is a consequence (for details see Sect. 4) of more or less similar shock speeds involved across the whole range of radial distances (Fig. 5b). Only a slightly decreasing trend of the overall $v(R)$ pattern can be noticed $(C=0.34)$. In average, the velocities decline from some $700 \mathrm{~km} \mathrm{~s}^{-1}$ at $R=2$ to about $500 \mathrm{~km} \mathrm{~s}^{-1}$ around $R=100$.

In Fig. $5 \mathrm{c}$ values of $B D W$ versus the inferred radial distance are shown. The overall trend shows a slight increase of $B D W(R)$, i.e. apparently the IP t-II bursts show somewhat larger values of $B D W$ than the metric ones. The $B D W$ distribution of the latter events is fairly 

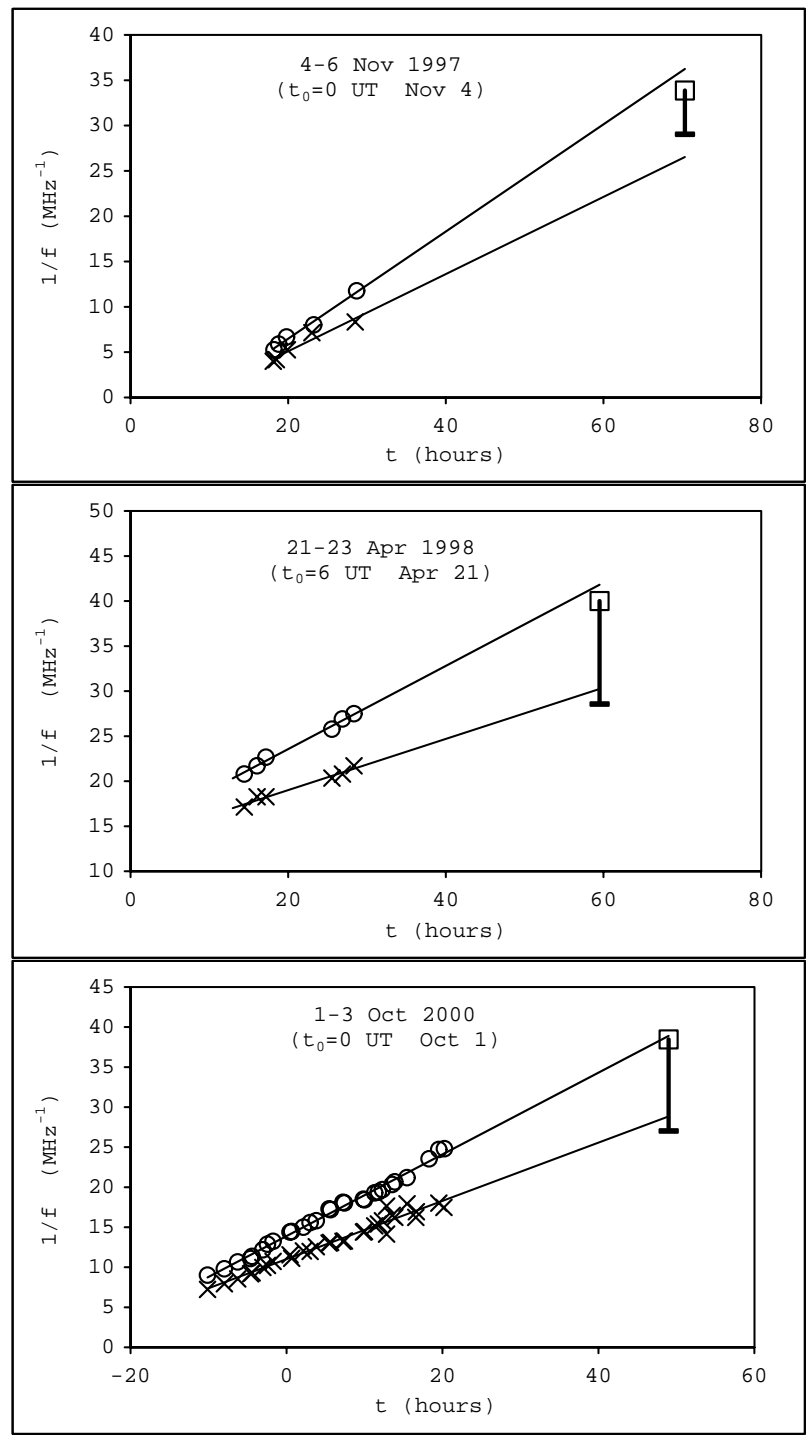

Fig. 4. Dynamic spectra represented as the inverse frequency $(1 / f)$ versus time $(t)$ for the events of: a) November 4-6, 1997; b) April 21-23, 1998; c) October 1-3, 2000. The low frequency branch (LFB) is shown by circles and the bold line, whereas the high frequency branch (HFB) is drawn by crosses and the thin line. The amplitude of the local frequency jump associated with the passage of shock front at $1 \mathrm{AU}$ is indicated by the vertical bold line (square marks the foot and bold dash the peak).

consistent with the results shown by Smerd et al. (1975). The values are somewhat smaller than in Mann et al. (1996), but it should be noted that bandwidths were determined therein using frequencies of "outer" borders of emission lanes and not of the centers as in here (see Fig. 2).

Figure 5c shows a void of data points in the region $2<R<4$ whose border is indicated by the (provisionally drawn) thin line. Let us note that in this range of radial distances the maximum of Alfvén speed is expected (Mann et al. 1999)

In Fig. 5d values of $B D W$ are drawn versus the inferred velocity $v$. Measurements embracing radial distances $R<10$ and $R>10$ are shown separately. In both
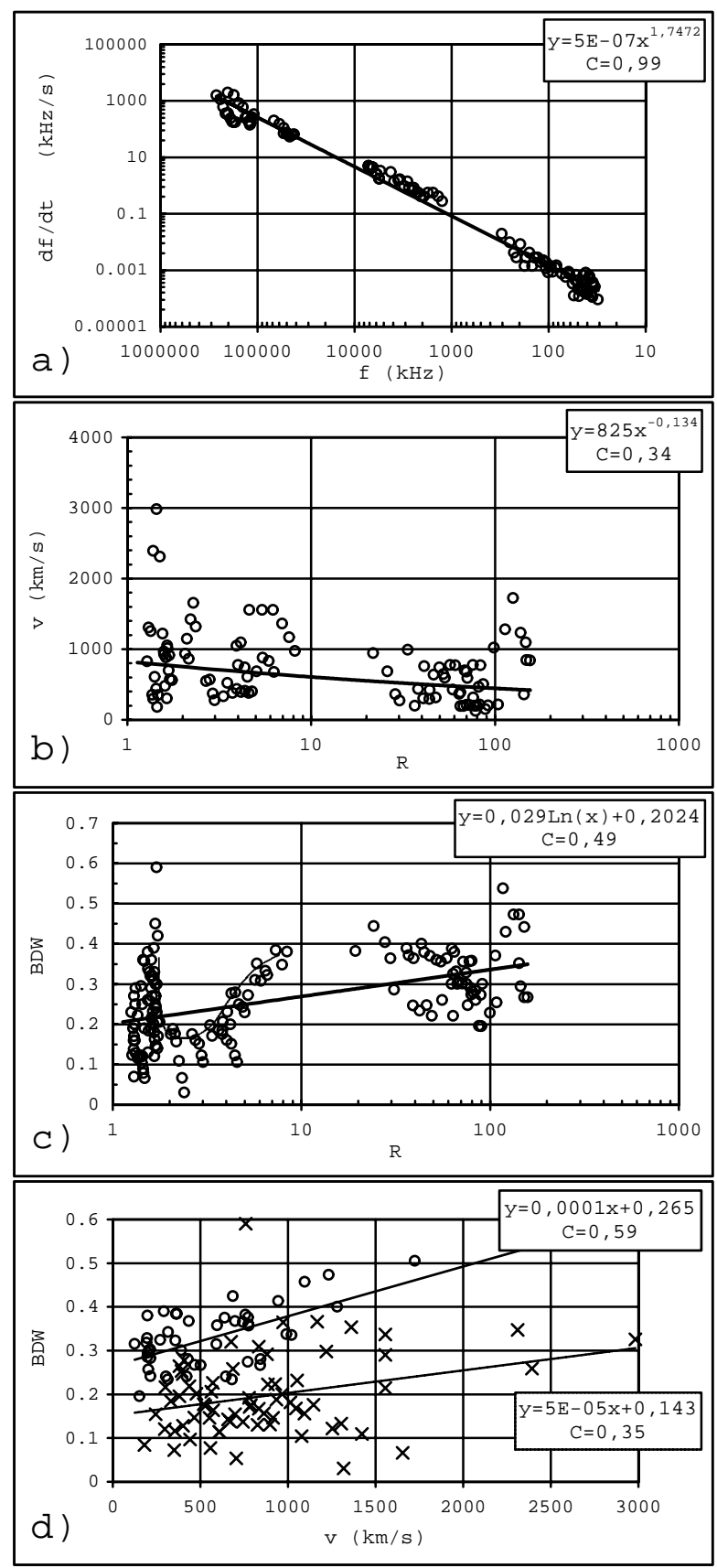

Fig. 5. a) Frequency drift shown versus frequency $\left(D_{f}(f)\right)$; b) Inferred velocity versus radial distance $(v(R))$; c) Emission split versus radial distance $B D W(R) ; \mathbf{d}) B D W$ versus inferred velocity. Crosses and thin line represent events at $R<10$, circles and bold line $R>10$.

subsamples $B D W$ shows in average larger values at higher velocities. The splits in the $R>10$ subsample are somewhat larger than in the $R<10$ subsample. Events cover more or less the same velocity range in both subsamples.

\section{Interpretation}

A synchronized frequency drift and similarity of intensity fluctuations of LFB and HFB suggest that these emission elements are coming from closely associated radio sources. 
On the other hand, in some particular IP t-II bursts the extrapolations of LFB and HFB point to the base and peak of the jump in the local plasma frequency caused by the passage of the associated shock. These two aspects of band-splitting indicate that LFB and HFB are emitted from the upstream and downstream region of the shock, as ad hoc presumed by various authors (see, e.g., Wild \& Smerd 1972; Smerd et al. 1974, 1975; Mann et al. 1995; Vršnak et al. 2001; and references therein).

Pre-examining a larger set of long wavelength IP t-II bursts we found in a number of non-band-split bursts that the emission lane points to the base of the associated jump in the local plasma frequency (indicative of the upstream emission) and sometimes (see Fig. 1c) to its peak (downstream emission). The former class of events and the events exposing the band-splitting (Fig. 4) show a disagreement with the conclusion drawn by Lengyel-Frey (1992) who inferred that the IP t-II emission comes preferably from the downstream region.

Accepting the interpretation in terms of upstream/downstream emission the value of $B D W$ can be related directly to the density jump across the shock. Since the plasma frequency is related to the electron density as $f \propto \sqrt{n}$ one finds:

$B D W=\frac{f_{2}-f_{1}}{f_{1}}=\frac{f_{2}}{f_{1}}-1=\sqrt{\frac{n_{2}}{n_{1}}}-1$,

where $n_{1}$ and $n_{2}$ are the electron densities in the upstream and downstream region, respectively. Using Eq. (4) the density jump can be expressed as:

$N_{12}=\frac{n_{2}}{n_{1}}=(B D W+1)^{2}$,

providing an estimate of the compression behind the shock front.

The density jump, which can be estimated using Eq. (5), is related to other shock characteristics (cf. Bazer \& Ericson 1959, see also Priest 1982). The bandsplitting thus provides valuable information about the shock. Among others, the limits for the magnetosonic Mach number $M$ can be estimated considering the quasiparallel and quasi-perpendicular propagation and assuming some reasonable values of the plasma to magnetic pressure ratio $\beta$. For example, in the case of the perpendicular shock in $\beta=0$ plasma, $B D W=0.5$ (i.e., $N_{12}=2.25$ ) corresponds to $M=M_{\mathrm{A}}=2.16$ where $M_{\mathrm{A}}$ is the Alfvén Mach number. Taking $\beta=1$ one finds $M=2.07$ and $M_{\mathrm{A}}=2.8$.

On the other hand, adopting some coronal or IP density model the frequency drift of t-II burst provides information on the shock velocity. Thus, the ambient Alfvén velocity and the magnetic field strength can be inferred (see, e.g., Smerd et al. 1975; Vršnak et al. 2001). This procedure will be applied in the forthcoming paper to map the Alfvén speed and the magnetic field from the corona to the Earth.

The examples shown in Figs. 1a, b, and 3b show a changing $B D W$ and $D_{f} / f$, revealing an evolution of the shock in a non-homogeneous environment. In general, the shock amplitude decreases if the ambient magnetosonic speed increases. In this sense the "void" in Fig. 5c at $2<$ $R<4$, where only low $B D W$ values are present, is most probably related to the Alfvén velocity increase towards the maximum that is expected at distances $3-4 R_{\odot}$ (Mann et al. 1999).

In particular, shocks generated from a "simple wave" (Mann 1995) decrease in amplitude also in the uniform medium due to the perturbation profile elongation (Landau \& Lifshitz 1987). So, metric t-II burst shocks formed from blast waves that are generated by flares (Vršnak \& Lulić 2000a,b) can hardly exceed the Alfvén speed maximum at $3-4 R_{\odot}$. This may explain why most metric t-II bursts, being excited by flare generated shocks (Magdalenić \& Vršank 2000; Vršnak 2001a) cease below the $20 \mathrm{MHz}$ layer (Nelson \& Melrose 1985). Consequently, IP t-II bursts are associated with CMEs (Cane et al. 1987) and generally are not related to metric t-II bursts (Gopalswamy et al. 1998).

In the IP space the Alfvén speed is decreasing. In such a situation it can be expected that the shock amplitude increases if the driver has an approximately constant velocity. This is consistent with the increasing trend of $B D W$ values in the IP space (Fig. 5c). A slight velocity decrease (Fig. 5b) might be reflecting the deceleration of fast CMEs (Gopalswamy et al. 2000; Vršnak 2001b) that drive the IP t-II burst shocks.

An approximately constant velocity results in the very good correlation of $D_{f}$ and $f$ revealed by Fig. 5a. Since the solar wind density behaves like $n \propto R^{-\alpha}$ after, say, $R \approx 0.1$ AU, where $\alpha \approx 2$ (Leblanc et al. 1998) one finds:

$D_{f} \equiv\left|\frac{\partial f}{\partial t}\right| \propto\left|\frac{\partial}{\partial t} \sqrt{n}\right| \propto\left|\frac{\partial}{\partial t} \frac{1}{R}\right| \propto \frac{v}{R^{2}} \propto n v \propto f^{2} v$

Adopting $v \approx$ const. Eq. (6) gives $D_{f} \propto f^{2}$. Using $v \propto$ $R^{-k}$ where $k \approx 0.1$ (see the inset in Fig. $5 \mathrm{~b}$ ) one finds $D_{f} \propto f^{1.9}$. We note that the fit regarding only IP events at $R>10$ really gives $k=1.9$ in the $D_{f}(f)$ power law fit, i.e. the value very close to the one expected for $n \propto R^{-2}$.

\section{Discussion}

The main shortcoming of the upstream/downstream interpretation is a need for the Langmuir turbulence in the downstream region since in situ measurements reveal that it is generally found in the upstream region of IP shocks (Lengyel-Frey et al. 1997; Thejappa \& MacDowall 2000). However, Thejappa \& MacDowall (2000) noted that none of the studied events was associated with the t-II emission and so is not representative for this subclass of shocks. On the other hand, Lengyel-Frey et al. (1992) reported electron plasma waves also in the downstream region.

Similarly, demand for the Langmuir turbulence in the downstream region faces theoretical problems (Treumann \& LaBelle 1992). Yet, some models predict Langmuir turbulence in the ramp of the shock (Ledenev 1996). 
Furthermore, one must bear in mind that the geometry of shock front and magnetic field lines is probably somewhat more complicated (Bale et al. 1999) than usually considered in the models. The t-II emission might be associated with the regions where field lines intersect the quasi-perpendicular shock twice, forming a collapsing trap geometry (see, e.g., Somov \& Kosugi 1997). Such a situation can arise either due to "bays" in the shock front (Bale et al. 1999; see also Zlobec et al. 1993; Vandas \& Karlický 2000) or at the locations where field lines are concave with respect to the shock front. The electrons can be accelerated several times in the reflections at the shock front, achieving large energies before being embraced by the shock. In such a configuration, Langmuir turbulence should be found in upstream as well as in the downstream region.

Alternative interpretations of band-splitting currently in use have even more severe deficiencies. For example, the interpretation proposed by Roberts (1959) based on the magnetic field effects (see also Tidman et al. 1966 and references therein) requires too large magnetic fields (Krüger 1979). As already mentioned in Sect. 2.1 the geometrical interpretation presuming a several-source emission (cf. McLean 1967) can hardly account for a synchronized intensity and frequency drift variations of LFB and HFB. On the other hand, it straightforwardly applies to the "multi-lane" type II bursts, where each component may show the band-splitting. The interpretation by Treumann \& LaBelle (1992) in terms of "caviton radiation" is limited to, say, $B D W<0.2$, whereas the measurements show $B D W$ up to 0.6 (see Fig. 5) or even higher (Mann et al. 1996). Likewise McLean's interpretation also cannot explain the relationship between the $B D W$ and the velocity (Fig. 5d) nor the tendency that extrapolations of LFB and HFB lanes point to the base and the ramp of the in situ shock signature (Fig. 4).

Finally, let us stress again that band-splitting can be found only in a fraction of t-II bursts (see Sect. 2.1). It is not a persistent phenomenon even within one event (especially in hekto-to-kilometric t-II bursts). Furthermore, as mentioned in Sect. 4.1 in some bursts the LFB is dominant, in other the HFB prevails, and sometimes the dominance changes in the course of the event. The question arises: what are conditions for the appearance of splitting?

First of all, the predominance of LFB or HFB should directly depend on the level of the Langmuir turbulence in the upstream and downstream regions, which can depend on local field/shock geometry. Furthermore the propagation effects can be different for LFB and HFB. These two items provide several possible explanations for the absence of band-splitting in large fraction of t-II bursts. If there is no collapsing trap geometry, and the level of Langmuir turbulence in the upstream region is high enough to produce the observable radio emission, only LFB will be observed. Another possibility is that electron plasma waves are present in both regions, but the LFB radio emission cannot escape and only HFB is observed. Finally, if the emission is weak, the absence of one component (i.e., the weaker emission branch) could be simply due to the sensitivity of measurements.

\section{Conclusion}

The band-split emission satisfying the criteria defined in Sect. 2.1 is usually attributed to metric t-II bursts. However, it can be found also in UC/IP t-II bursts. The investigation of band-splitting presented in this paper shows that it can be consistently interpreted in terms of the emission from the upstream and downstream shock region. So, the measurements of $B D W$ and $D_{f}$ can give a valuable information about the MHD shock evolution in the nonhomogeneous magnetoplasma. Such measurements are potentially also an important diagnostic tool for mapping the coronal and IP magnetic fields. This can be of special interest in investigating regions like the upper corona where other diagnostic methods are not applicable.

Finally, let us stress another significant implication of the upstream/downstream emission interpretation. When the radial distance of the radio source is to be inferred from the frequency of emission, the LFB should be used, i.e. the upstream (unperturbed) plasma frequency. For example, let us take $B D W=0.5$ at the emission frequency of $f_{1}=$ $1000 \mathrm{kHz}\left(f_{2}=1500 \mathrm{kHz}\right)$. Applying the density model by Leblanc et al. (1998) and using $f_{1}$ one finds $R_{1}=6.27$. Erroneously using $f_{2}$ one would find the distance of only $R_{2}=4.84$. This can be a considerable difference when the position of the radio source is to be compared with the location of, e.g., the leading front of a CME. Similarly, the velocities would be underestimated using $f_{2}$ instead of $f_{1}$. For example, in the case of IP t-II burst shown in Fig. 1c one would obtain the value of $450 \mathrm{~km} \mathrm{~s}^{-1}$ instead of the correct speed of $550 \mathrm{~km} \mathrm{~s}^{-1}$.

\section{References}

Bale, S. D., Reiner, M. J., Bougeret, J.-L., et al. 1999, Geophys. Res. Lett., 26, 1573

Bazer, J, \& Ericson, W. B. 1959, ApJ, 129, 758

Boischot, A., Riddle, A. C., Pearce, J. B., \& Warwick, J. W. 1980, Sol. Phys., 65, 397

Bougeret, J. L., et al. 1995, Space Sci. Rev., 71, 231

Bougeret, J. L. 1985, in Collisionless Shocks in the Heliosphere, Geophys. Monograph 35 (AGU), 13

Cane, H. V., Stone, R. G., Fainberg, J., Steinberg, J. L., \& Hoang, S. 1982, Sol. Phys., 78, 187

Cane, H. V., Sheeley, N. R., \& Howard, R. A. 1987, J. Geophys. Res., 92, 9869

Gopalswamy, N., et al. 1998, J. Geophys. Res., 103, 307

Gopalswamy, N., Lara, A., Lepping, R. P., et al. 2000, Geophys. Res. Lett., 27, 145

Krüger, A. 1979, Introduction to Solar Radio Astronomy and Radio Physics (Reidel, Dordrecht)

Landau, L. D., \& Lifshitz, E. M. 1987, Fluid Mechanics, 2nd Ed. (Pergamon Press, Oxford)

Ledenev, V. G. 1996, A\&A, 316, 435 
Lengyel-Frey, D., \& Stone, R. G. 1989, J. Geophys. Res., 94, 159

Lengyel-Frey, D. 1992, J. Geophys. Res., 97, 1609

Lengyel-Frey, D., MacDowall, R. J., Stone, R. G., et al. 1992, in Solar Wind Seven, ed. E. Marsch, \& R. Schwenn (Pergamon Press, Oxford), 477

Lengyel-Frey, D., Thejappa, G., MacDowall, R. J., Stone, R. G., \& Phillips, J. L. 1997, J. Geophys. Res., 102, 2611

Leblanc, Y., Dulk, G. A., \& Bougeret, J.-L. 1998, Sol. Phys., 183,165

Magdalenić, J., \& Vršnak, B. 2000, Hvar Obs. Bull., 24, 1

Mann, G. 1995, J. Plasma Phys., 53, 109

Mann, G., Classen, T., \& Aurass, H. 1995, A\&A, 295, 775

Mann, A., Aurass, H., Voigt, W., \& Paschke, J. 1992, ESA SP-348, 129

Mann, G., Klassen, A., Classen, T., et al. 1996, A\&AS, 119, 489

Mann, G., Aurass, H., Klassen, A., Estel, C., \& Thompson, B. J. 1999, ESA SP-446, 477

McLean, D. J. 1967, Proc. ASA, 1, 47

Nelson, G. S., \& Melrose, D. B. 1985, in Solar Radiophysics, ed. D. J. McLean, \& N. R. Labrum (Cambridge Univ. Press, Cambridge), 333

Newkirk, G. Jr. 1961, ApJ, 133, 983

Priest, E. R. 1982, Solar magnetohydrodynamics (Reidel Publ. Co., Dordrecht)
Reiner, M. J., Kaiser, M. L., \& Bougeret, J.-L. 2001, J. Geophys. Res., in press

Roberts, J. A. 1959, in Paris Symposium on Radio Astronomy, ed. R. N. Bracewell (Stanford Univ. Press), 194

Saito, K. 1970, Ann. Tokyo Astr. Obs., 12, 53

Smerd, S. F., Sheridan, K. V., \& Stewart, R. T. 1974, in IAU Symp. 57, ed. G. A. Newkirk, 389

Smerd, S. F., Sheridan, K. V., \& Stewart, R. T. 1975, ApL, 16,23

Somov, B. V., \& Kosugi, T. 1997, ApJ, 485, 859

Thejappa, G., \& MacDowall, R. J. 2000, ApJ, 544, L163

Tidman, D. A., Birmingham, T. J., \& Stainer, H. M. 1966, ApJ, 146, 207

Treumann, R. A., \& LaBelle, J. 1992, ApJ, 399, L167

Vandas, M., \& Karlický, M. 2000, Sol. Phys., 197, 85

Vršnak, B. 2001a, J. Geophys. Res., in press

Vršnak, B. 2001b, Sol. Phys., in press

Vršnak, B., \& Luli'c, S. 2000, Sol. Phys., 196, 157

Vršnak, B., \& Luli'c, S. 2000, Sol. Phys., 196, 181

Vršnak, B., Magdalenić, J., \& Aurass, H. 2001, Sol. Phys., in press

Weiss, A. A. 1963, Aust. J. Phys., 16, 240

Wild, J. P., \& Smerd, S. F. 1972, ARA\&A, 10, 159

Zlobec, P., Messerotti, M., Karlický, M., \& Urbarz, H. 1993, Sol. Phys., 144, 373 